\title{
Intervención Formativa con el Profesorado de Danza e Influencia Motivacional en su Alumnado
}

\author{
Formative Intervention with Dance Teachers \\ and Motivational Influence in their Students
}

\section{Intervençáo formativa em professores de dança e a influencia da motivaçáo nos alunos}

\author{
Ana García-Dantas ${ }^{1}$ José Carlos Caracuel-Tubío $^{1}$ y Rafael Peñaloza-Gómez ${ }^{2}$ \\ ${ }^{1}$ Universidad de Sevilla, España, ${ }^{2}$ Universidad Autónoma Nacional de México
}

\begin{abstract}
Resumen: Se diseńa un programa de formación para el profesorado con el principal objetivo de evaluar si sus estudiantes mejoran sus niveles motivacionales. Integraron la muestra 5 profesores voluntarios de un Conservatorio de danza (3 del grupo experimental y 2 del grupo control), así como sus respectivos alumnos $(\mathrm{N}=60)$. Se evaluó antes de la intervención la conducta de instrucción mediante el CBAS (Coaching Behaviour Assessment System) adaptado a danza, así como la motivación de los alumnos a través de un Cuestionario de Motivación para Estudiantes de Danza. Después del trabajo con profesores del grupo experimental, se les evaluó de nuevo para comprobar si los niveles iniciales reflejaban algún cambio. Los resultados muestran variaciones significativas en las escalas del CBAS y en los cuestionarios de Motivación en dicho grupo sugiriendo la efectividad de la intervención.

Palabras clave: Motivación, danza, intervención, bailarines, conservatorio, CBAS.

Abstract: An educational programme for teachers was designed with the aim to improve their students' motivation. A sample consisting of five teachers from a dance conservatoire (experimental group $n=3$, control group $\mathrm{n}=2)$, and their respective students $(\mathrm{N}=60)$ took part in this study. The behavior of the teachers was assessed using the adapted Coaching Behavior Assessment System (CBAS).The students' motivation was assessed using the "Motivation questionnaire for dance students [Cuestionario de Motivación
\end{abstract}

para Estudiantes de Danza]". Following the intervention, teachers and students in the experimental group were assessed using the instruments listed previously to observe any changes in motivation between pre and post test. Results showed significant differences in scales of CBAS and the questionnaires from pre to post test in the experimental group. The control group, however, did not display significant differences, thus suggesting the intervention was effective.

Keywords: Motivation, dance, intervention, dancers, conservatoire, CBAS Resumo: Foi desenhado um programa de formação para os professores com o principal objetivo de avaliar se os estudantes melhoraram os níveis de motivação. A mostra foi composta por 5 professores voluntários de um Conservatório de Dança ( 3 do grupo experimental e 2 do grupo controle), e seus respectivos alunos $(\mathrm{N}=60)$. Foi avaliada a conduta de instrução antes da intervenção, através do CBAS (Coaching Behaviour Assessment System) adaptado á dança, e também a motivaçáo dos alunos através do Questionário de Motivação para Estudantes de Dança. Depois do trabalho com os professores do grupo experimental, novamente foi avaliado para comprovar se os níveis iniciais apresentavam alguma alteraçáo. Os resultados mostram variaçôes significativas nas escalas do CBAS e no questionário de Motivação no grupo referido, sugerindo a efetividade da intervenção.

Palavras Chave: Motivação, dança, intervençẫo, bailarinas, conservatório, CBAS.
El ejercicio general de la docencia -indistintamente del tipo y nivel de la misma- encuentra problemas comunes, especialmente en lo que hace a la interacción docente-discente. Algunos de esos problemas tienen que ver con aspectos mo-

Dirección para correspondencia [Correspodence address]: Ana GarcíaDantas,

E-mail: anagarciadantas@gmail.com Dirección: Departamento de Personalidad, Evaluación y Tratamiento psicológicos, Facultad de Psicología, Universidad de Sevilla. c/ Camilo J. Cela, s/n. 41018 Sevilla (España).

Agradecimientos al Conservatorio Profesional de Danza de Sevilla "Antonio Ruiz Soler". tivacionales, tanto del profesorado como de los estudiantes, problemas que, de no ser tratados adecuadamente, pueden desembocar en el abandono -a veces prematuro- de una parte significativa del alumnado. Uno de ellos parece ser el estilo del docente a la hora de impartir enseńanza e interactuar con su grupo de clase.

En los últimos años, los avances que han tenido lugar sobre la motivación en el campo de la educación han establecido ciertas pautas que sirven al profesorado, en muchas ocasiones, de guía para el funcionamiento como docente en las clases. Numerosos estudios han resaltado la relevancia que tiene la figura del profesorado a la hora de establecer una relación 
positiva con el alumnado, la mejora de la motivación de éste último, la cantidad de logros conseguidos, así como las actitudes de aprendizaje que presentan (Day, Elliot \& Kington, 2005; Firestone, 1996; Graham, 1996; Louis 1998). En el terreno de la danza, el estudio de la motivación se hace incluso más patente debido a la escasez de investigaciones que existen y al estricto y duro trabajo al que los bailarines se enfrentan diariamente a lo largo de su vida profesional.

Según Taylor y Taylor (2008), tanto los aprendices como los profesionales de la danza muy motivados, se caracterizan por un elevado nivel de persistencia en el entrenamiento y una destacada capacidad para persistir ante el aburrimiento, la fatiga, el dolor o el deseo de hacer otras cosas. De hecho, los bailarines y bailarinas más motivados dedican más tiempo y esfuerzo a mejorar su rendimiento. Entre las características más importantes que inducen a considerar que un bailarín presenta altas dosis de motivación se encuentran: a) alto entusiasmo en clase y en los ensayos; b) elevado interés por aprender pasos y técnicas nuevas que conduzcan a la mejora de la ejecución; c) gran adherencia a la actividad siendo los/as primeros/as que llegan a clase y de los últimos en marcharse; d) mayor inversión de tiempo fuera del entrenamiento para trabajar y practicar lo aprendido en éste; e) mayor deseo por seguir ensayando a pesar de estar enfermos y con dolencias; y f) mejor planteamiento de objetivos, siendo éstos claros y específicos.

Analizadas las principales facetas de los bailarines que conllevan altas dosis de motivación, es lógico preguntarse por todos aquellos factores que, de una forma u otra, promueven y facilitan su mejora. Desde hace más de una década, ya se intuían las características principales que todo profesor o profesora de danza debía tener para que su alumnado rindiera más. Thomas (1993), enunció las bases que se debían seguir en una clase de danza. De una forma resumida, resaltaba las siguientes: el profesorado de danza verdaderamente efectivo es muy positivo; muestra apoyo y entusiasmo; suministra al alumnado feedback en una terminología positiva; se focaliza en el futuro y no el pasado; controla el ejercicio a través del consenso y no de la coerción; hace frente a los problemas desde edades tempranas antes de que se conviertan en problemas reales e, intuitivamente, entiende y maneja las dinámicas sociales.

De forma paralela, en el campo del deporte se han realizado numerosos estudios con el objetivo de examinar la dependencia entre las conductas del entrenador y la percepción de la motivación en los deportistas. Entre ellos cabe destacar el de Black y Weiss (1992), con una muestra de jóvenes nadadores. Los resultados obtenidos revelaron que las autopercepciones de los atletas y su motivación, estaban estrechamente relacionadas con la cantidad y calidad de la retroalimentación recibida por parte del entrenador ante situaciones deportivas destacadas o rendimientos no deseados.
Amorore y Horn (2000), siguiendo la misma línea, encontraron que los entrenadores percibidos como otorgando altas frecuencias de retroalimentación positiva, ánimo e información y bajas frecuencias de las conductas que ignoraban el rendimiento de los atletas, tenían deportistas que puntuaban niveles más altos de motivación intrínseca.

Es importante, por tanto, si se pretende intervenir en este terreno, evaluar con precisión las conductas del profesorado, ya que es habitual que en ocasiones, ellos sobreestimen el número de conductas reforzantes, así como la calidad de sus instrucciones. De hecho, así lo demuestra el estudio de Wandzilak y Ansorge (1998), en el cual se concluye que los entrenadores participantes pensaban que animaban en un porcentaje mayor de lo que realmente lo hacían, destacando la necesidad de ayuda que estas figuras tienen para ser más conscientes de lo que hacen, a fin de favorecer su interacción como entrenadores.

Rodríguez (2010), llevó a cabo un trabajo de profundización sobre los estudios relacionados con el tema, basados en los principios del modelo mediacional y del sistema de observación conductual CBAS (Smith, Smoll y Hunt, 1977). De este modo, Rodríguez enumeran las principales conclusiones encontradas respecto a la relación existente entre las conductas de los entrenadores y las reacciones de los atletas hacia su práctica deportiva:1) El entrenador juega un rol crucial en la calidad de la experiencia deportiva de los jóvenes; 2) Los jugadores respondían más favorablemente a los entrenadores que utilizaban mayores porcentajes de conductas de apoyo e instrucción; 3) Los entrenadores que otorgaban altos niveles de reforzamiento hacia los rendimientos deseados e instrucciones técnicas ante los errores, eran muy valorados por los estudiantes; 4) El agrado mostrado por los jugadores hacia su entrenador no se encontraba relacionado con el registro de victorias y derrotas obtenidas por el equipo.

Conocidas las ventajas de un entrenamiento con alta frecuencia de conductas de apoyo e instrucción y bajo nivel de punición, se ha procurado promover un estilo favorable de entrenamiento que predisponga una adecuada situación de aprendizaje en contextos deportivos. Sin embargo, la tradicional concepción de la enseńanza de la danza, estaba plenamente dirigida por el profesorado y centrada en su autoridad mientras que el alumnado mostraba una actitud pasiva a la vez que la recibía. Esta docencia entendida por estudiantes, y profesores, y por la sociedad en general, como una verdad universal e incuestionable (Marrero, 1993), podría estar frenando de alguna manera, el surgimiento de estudios que resalten la importancia del alumnado en escuelas de danza y conservatorios.

Se ha encontrado además, que los bailarines presentan algunas conductas perjudiciales para la salud típicamente, como trastornos de la conducta alimentaria (Ackard, Henderson y Wonderlich, 2004), abuso de sustancias (Sekulic, 
Peric, Rodek, 2010) o elevados índices de ansiedad (Gordon, 2005). El profesorado, por lo general no está preparado para prevenir, tratar o derivar en su caso, ni éstos ni otros problemas que surgen en las clases. En relación con esto, buena parte de las investigaciones que se llevan a cabo en el terreno de la danza coinciden en concluir la necesidad del estudio de los fenómenos que afectan a los bailarines y a su entorno, ya sea en el nivel de iniciación como en el profesional y de formar al profesorado para que pueda actuar en consecuencia (Carter, 2005; Chappell, 2007; Sekulic, Peric \& Rodek, 2010).

En muchos lugares, el trabajo psicológico con los bailarines dentro de los Conservatorios y escuelas de danza -tanto a nivel amateur como profesional- es prácticamente inexistente; a pesar de ello, López de la Llave y Pérez-Llantada (2006), pioneros en el campo de la Psicología de la interpretación artística en nuestro país, muestran algunas directrices de enseńanza basadas en las Leyes del Aprendizaje, bajo las cuales consideran que un intérprete joven (bailarín/a, músico/a o actriz/actor) obtendrá mayor provecho de las clases y, consecuentemente, rendirá mejor.

Sin embargo, hasta donde se ha podido encontrar, se carece de estudios de campo e investigaciones en danza en nuestro país que informen sobre el estilo docente que promueve una mayor satisfacción y rendimiento en el alumnado, siendo esta actividad artística considerada como una disciplina con peculiaridades diferentes en muchos sentidos al deporte que tradicionalmente es estudiado, como el fútbol o la natación. (Caracuel \& García-Dantas, 2010; García-Dantas \& Caracuel, 2011). De este modo, tal y como concluyen Brinson (1993) y Brinson y Dick (1996), es probable que la intervención y la promoción de ciertas actitudes y conductas en este campo, produzcan considerables mejoras después de trabajar con este colectivo con frecuencia abandonado.

El presente estudio surge a raíz de los resultados de una investigación previa realizada en el Conservatorio Profesional de Danza "Antonio Ruíz Soler" de Sevilla (España), a partir de la cual se comprobaron las relaciones existentes entre ciertos aspectos psicológicos como la motivación o el nivel de competencia percibida y el abandono definitivo de la actividad (García-Dantas y Caracuel, 2009; 2010). El análisis de la situación estimó conveniente el trabajo motivacional con el profesorado con el objetivo general de identificar el estilo docente que produce mayor motivación en el alumnado y a la vez, 1) formar al profesorado en técnicas psicológicas que promuevan un mejor rendimiento y un clima de trabajo más adecuado en las clases; 2) evaluar el nivel motivacional del alumnado; y 3) comprobar la eficacia de las técnicas psicológicas impartidas.

Para tratar de cumplir dichos objetivos se plantearon como hipótesis iniciales del estudio las siguientes:

1.- Las conductas observadas de Apoyo e Instrucción de los profesores del grupo experimental mejorarán en fre- cuencia tras la intervención psicológica (formación) y no lo harán en el grupo control.

2.- Las conductas Punitivas del profesorado del grupo experimental disminuirán tras la formación y no así las del grupo control.

3.- El valor general referido a la motivación del alumnado aumentará tras la formación que recibirán los profesores, cambiando positivamente en los tres aspectos motivacionales estudiados: percepción del estilo del profesorado, sensación propia motivacional en sus clases y relevancia otorgada a la motivación para el buen rendimiento y satisfacción del bailarín/a.

\section{Método}

\section{Participantes}

\section{a) Profesorado}

Colaboraron en el estudio de forma voluntaria 5 profesores/as del Conservatorio Profesional de Danza de Sevilla "Antonio Ruíz Soler". Tres de los profesores participantes acudieron a las sesiones formativas y se beneficiaron de la intervención; los otros dos no pudieron por razones personales, por lo que se utilizaron sus datos como control del estudio. Cada profesor/a fue evaluado en función de la relación concreta que mantenía con un grupo específico y no así con todo su alumnado en general. El Grupo Experimental (GE) lo integraron tres profesores, un varón y dos mujeres; todos impartían clases en diferentes cursos de la espacialidad de danza española (40 de Enseñanzas Básicas, $1^{\circ}$ y $4^{\circ}$ de Grado Profesional) enseñando asignaturas de espańol, estilizada y repertorio. El Grupo Control (GC) estuvo integrado por dos profesores, un varón y una mujer; el primero, profesor de danza contemporánea, impartía clases a $1^{\circ}$ de Grado Profesional de contemporáneo; la segunda, profesora de danza clásica, en $1^{\circ}$ de Grado Profesional de flamenco. Sus edades oscilaban entre 30 y 45 años.

El método de selección de la muestra fue intencional a partir de todo el profesorado que se presentó voluntario para participar en el estudio. El criterio que se siguió pretendía contar con diferentes cursos, especialidades, sexo y asignaturas. Se excluyó a una profesora del GE del estudio debido a una baja por lesión en el momento de la recogida postest de los datos, con lo que el GE-Profesores quedó integrado por tres personas.

\section{b) Alumnado}

Participaron 60 estudiantes en total distribuidos por grupo, sexo, curso y especialidad tal y como aparece en la Tabla 1 . La mortandad de la muestra con respecto a la segunda recogida de datos fue de 9 participantes. 
Tabla 1. Distribución Porcentual y de Frecuencia de todo el alumnado que participó en el pretest según el Grupo al que pertenecían, el Sexo, el Curso y su Especialidad

\begin{tabular}{cccc}
\hline Grupo & Sexo & Curso & Especialidad \\
\hline Experimental & Varones & $4^{\circ}$ E.B. & Contemporáneo \\
$45(75 \%)$ & $6(10 \%)$ & $22(36,7 \%)$ & $7(11,7 \%)$ \\
& & & \\
Control & Mujeres & $1^{\circ}$ G.P. & Español \\
$15(25 \%)$ & $54(90 \%)$ & $23(38,3 \%)$ & $45(75 \%)$ \\
& & $4^{\circ}$ G.P. & Flamenco \\
& & $12(25 \%)$ & $8(13,3 \%)$ \\
\hline
\end{tabular}

Nota: E.B.: Enseñanzas Básicas; G.P.: Grado Profesional

\section{Instrumentos}

- Para evaluar distintos factores motivacionales, se aplicó el Cuestionario de Motivación para Estudiantes de Danza, creado expresamente para esta ocasión. Dicho cuestionario se compone de 15 ítemes a contestar en una escala tipo Likert de 1 (nada de acuerdo) a 5 (totalmente de acuerdo). Se comprobó la pertinencia del análisis factorial del instrumento a partir de tres pruebas: a) los coeficientes de correlación de Pearson entre determinados ítemes reflejan valores altamente signi- ficativos; b) los índices KMO -Kaiser-Meyer-Olkin (.805) se consideran aceptables, esto es cuando superan .06 y c) la prueba de esfericidad de Barlett presenta una Chi-cuadrado aproximada de 601,05 y una significación de .000. Como se deduce de estos datos, las correlaciones entre los ítemes presentan relaciones estadísticamente significativas y, por tanto, es pertinente el análisis factorial para conocer la agrupación entre las preguntas. El programa señala 3 factores principales que explican el 53,67 \% de la varianza de las respuestas a los ítemes gracias al método de extracción de componentes principales.

El gráfico de sedimentación, cuya función es representar los factores y los autovalores asociados, confirma dicha división. Por último, la matriz de componentes rotados para analizar las saturaciones de los ítemes -esto es, en el caso de las soluciones ortogonales, la correlación entre un ítem y un factor- ha demostrado ser superior a .30 para todos los ítemes, exceptuando al 14 y el 15. El índice de fiabilidad de los 15 ítemes que componían inicialmente el cuestionario posee un Alpha de Cronbach de .80, considerado un nivel alto de fiabilidad. En la Tabla 2 se observan dichas escalas con los ítems correspondientes.

Tabla 2. Distribución de Ítemes por Factor según Análisis Factorial Exploratorio del Cuestionario de Motivación para Estudiantes de Danza.

Factor 1

Estilo del profesor

2 Mi profesor/a nos motiva para que disfrutemos aprendiendo la técnica

3 Imparte unas clases amenas que rompen con la monotonía de las demás

4 Promueve un buen clima de trabajo en clase

5 Mi profesor/a me reconoce las cosas cuando las hago bien

$6 \mathrm{Mi}$ profesor/a sabe apreciar lo positivo de todos/as nosotros/as

9 Tengo buena comunicación con mi profesor/a

10 Sé que cuento con su ayuda ante cualquier problema

$11 \mathrm{Mi}$ profesor/a nos comprende y se preocupa por nuestro bienestar

Factor 2

Sensación propia

1 Vengo con ganas a recibir esta clase

7 Me gusta el método de enseñanza que utiliza

8 Mi profesor/a se esfuerza por tratarnos a todos/as por igual

Factor 3

Interacción motivacional

12 Es importante que los alumnos/as nos sintamos motivado/as en las clases

13 Cuando no estoy motivado/a no aprovecho la clase como debería

14 Un bailarín/a motivado /a rendirá mejor que otro que no lo está

15 Es importante que los profesores/as sepan cómo motivarnos

Los objetivos del cuestionario se corresponden con dichos factores, con los que se persigue evaluar la percepción del alumnado sobre: a) el estilo del profesor/a al dar las clases, b) la sensación motivacional que perciben de este estilo, y c) la importancia que otorgan al papel de la motivación en danza (denominado interacción motivacional).

- El registro observacional del profesorado se llevó a cabo mediante el CBAS (Coaching Behavior Assessment System, Smith, Smoll y Hunt, 1977), adaptado por los autores al contexto de la Danza (ver ANEXO). Este instrumento evalúa las 
interacciones básicas y de comunicación entre el entrenador/a y sus pupilos en una situación deportiva, en este caso de un profesor/a en una situación de danza. Se pueden identificar en el CBAS adaptado 3 dimensiones conductuales principales (Rodríguez, 2010) compuestas por los siguientes factores:

1.) Apoyo: conductas de reforzamiento, ánimo contingente al error, ánimo general y desánimo/desgana (puntuación negativa).

2.) Instrucciones: instrucciones técnicas generales e instrucciones técnicas contingente al error.

3.) Punición: castigo o punición, instrucciones técnicas punitivas y reprimendas.

- Las técnicas motivacionales para el profesorado de danza fueron diseñadas especialmente para esta ocasión, así como las actividades que se realizaron durante las sesiones formativas. Dichas sesiones fueron diseñadas combinando estrategias de corte conductual y constructivistas a través de las cuales se trabajaban técnicas que fomentaban la motivación del alumnado. Las sesiones se diseñaron con el objetivo de tener un espacio donde entrenar las habilidades aprendidas con los compañeros, así como de ponerlas en práctica en las clases y comentarlas en la siguiente sesión. La promoción de alternativas como sustitutas de las conductas punitivas fue esencial durante las tres sesiones formativas, especialmente el uso del reforzamiento positivo como técnica para incrementar las conductas deseadas.

\section{Procedimiento}

El estudio se llevó a cabo gracias a la participación del profesorado voluntario que accedió a colaborar en el programa de motivación en el Conservatorio Profesional de Danza "Antonio Ruíz Soler". Las fases en las que se dividió el estudio fueron las siguientes:

1.- Evaluación pretest. Se llevaron a cabo las siguientes actividades: a) realización del registro conductual (CBAS adaptado) de cada profesor/a en interacción con su grupo-clase durante dos sesiones de 40 minutos cada una; b) aplicación del Cuestionario de Motivación para Estudiantes de Danza en dicho grupo.

2.- Intervención: Consistió en tres sesiones -de dos horas cada una- de charlas motivacionales para el profesorado. Entre éste, se encontraban tres de los cinco participantes del estudio y otros más que no fueron evaluados. Las sesiones se impartieron alternando una semana sin charlas y la siguiente con charlas, a fin de dejar un tiempo de reflexión y de puesta en práctica de lo aprendido.

3.- Evaluación post-test: Al cabo de un mes se llevó a cabo: a) un nuevo registro conductual de los profesores/as, tanto a aquellos tres que habían asistido (grupo expe- rimental), como a los que no (grupo control), con sus mismos grupos de clase y durante el mismo tiempo; b) una nueva aplicación del Cuestionario de Motivación para Estudiantes de Danza, referente a cada profesor/a en cuestión.

Se considera como Variable Independiente la asistencia a las sesiones formativas y como Variables Dependientes el registro conductual del profesorado así como la motivación del alumnado, obtenida gracias al citado cuestionario.

El período total de intervención y evaluación comprendió 4 meses aproximadamente (Febrero-Mayo). Con el objetivo de modificar al mínimo las condiciones naturales en las que este colectivo se desenvuelve diariamente; la evaluación y las charlas se llevaron a cabo en las aulas habituales donde se ensaya.

Los cuestionarios se aplicaron antes de empezar la clase, cuando el profesor aún no había llegado; se solicitaba a los alumnos y alumnas que para respetar la confidencialidad de las respuestas de los compañeros se separaran unos de otros $y$, aunque no se les informaba sobre el objetivo del estudio, se les explicaba la importancia de la sinceridad así como del anonimato de los resultados. Cuando se procedía al registro conductual del profesorado se les informaba a todos/as que no se trataba de ningún examen práctico y que debían comportarse de la forma más similar posible a un día cualquiera de entrenamiento.

\section{Análisis de datos}

El análisis de los datos se llevó a cabo mediante el programa estadístico SPSS 17. En primer lugar se calculó la fiabilidad del cuestionario y se realizó un análisis factorial exploratorio. Para evaluar si existían cambios entre los datos recogidos (tanto de profesores como de alumnos) antes y después de la intervención, se realizó un análisis de diferencia de pruebas relacionadas.

\section{Resultados}

Este estudio fue realizado sobre una muestra de profesores pertenecientes a diversas especialidades, distintos cursos y diferente sexo del Conservatorio Profesional de Danza de Sevilla. Por contra, la edad de los mismos fue lo más homogénea posible. Todo ello a fin de garantizar la representatividad de la muestra.

El primer propósito de esta investigación fue comprobar si la impartición de sesiones de formación sobre motivación a un grupo de profesores producía cambios en su estilo docente. Para ello se utilizó el sistema de registro observacional CBAS adaptado (ver descripción del instrumento) a fin de evaluar las conductas correspondientes a las categorías de Instrucción, 
Apoyo y Punición antes y después de la intervención. Los resultados muestran que realmente hubo el cambio esperado, aumentando la frecuencia de Instrucciones y Apoyo y reduciéndose las de Punición.

Los datos de la prueba de medias para el Grupo experimental, nos indican que hubo diferencias estadísticamente significativas en la dimensión de Instrucciones $(t(2)=-15.5$; $\left.\mathrm{p}=.004 ; \mathrm{n}^{2}=.99\right)$ aumentando la frecuencia de instrucciones en el post-test $(M=13,66)$ en comparación con el pre-test $(M=6,83)$. En el caso del Apoyo no se encontraron diferencias estadísticamente significativas $t(2)=-4,16, \mathrm{p}=.053 ; \mathrm{n}^{2}=$
.90 aunque sí existía una tendencia clara positiva de mejora entre el antes de las sesiones $(M=2,41)$ y el después $(M=6,50)$. La variable denominada como Punición $t(2)=1.98 ; \mathrm{p}=.189$ no presentó diferencias estadísticamente significativas entre los datos del pre-test $(M=6,44)$ y del post-test $(M=0,88)$; no obstante el descenso de la frecuencia de los castigos y reprimendas después de las sesiones de intervención es llamativo. En la Figura 1 se muestran las puntuaciones obtenidas por los profesores del Grupo Experimental en las tres dimensiones mencionadas, apreciándose los cambios en las mismas del pretest al postest.

Figura 1. Frecuencia total de conductas registradas a través del CBAS del profesorado perteneciente al Grupo Experimental antes y después de las sesiones.

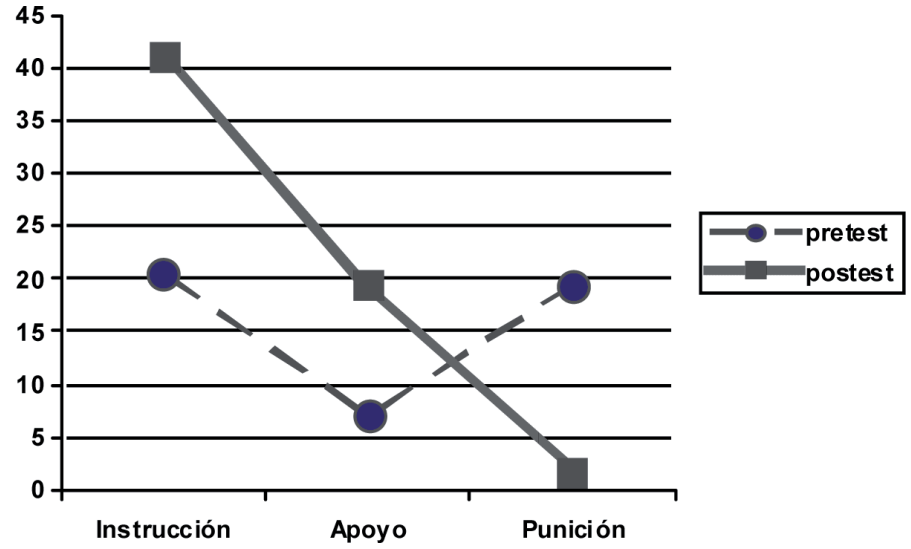

Para corroborar que los cambios en el Grupo Experimental se debían a las sesiones formativas impartidas, se utilizó un Grupo Control de referencia. En la variable Instrucciones los dos profesores analizados presentaron un aumento en sus puntuaciones en el postest. En la dimensión de Apoyo, en el caso del profesor 4 la puntuación media disminuyó del pre- test al post-test, mientras que en el Profesor 5 aumentó; con respecto a la Punición sólo el Profesor 5 disminuyó sus puntuaciones en el Post-test en comparación con el Profesor 4. Los cambios en estas dos últimas dimensiones no parecen seguir un patrón fijo ni regular por lo que no parecen ser debidos la influencia de alguna variable controlable (ver Figura 2). 
Figura 2. Frecuencia total de conductas registradas a través del CBAS del profesorado perteneciente al grupo control en dos momentos puntuales del curso correspondientes con el pretest y postest del grupo experimental.

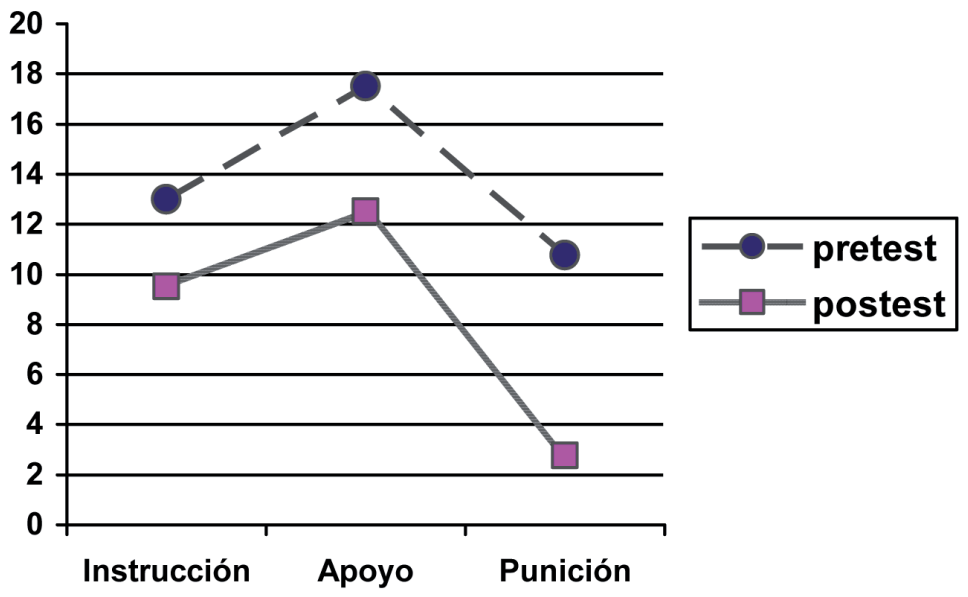

Para comprobar si existían diferencias significativas se realizó un análisis de medias para muestras relacionadas en las puntuaciones antes y después de la intervención de los profesores del Grupo Control; los resultados obtenidos no muestran diferencias estadísticamente significativas en ninguna de las dimensiones: Instrucciones $t(1)=-5.0 ; \mathrm{p}=13$; Apoyo $t(1)=.071$; $\mathrm{p}=.955 ; \mathrm{y}$ Punición $t(1)=1.0 ; \mathrm{p}=.50$.

Como se ha podido comprobar, las sesiones formativas ejercieron el efecto pretendido sobre los profesores que las recibieron, aumentando la frecuencia de la categoría Instrucciones y Apoyo y disminuyendo la de Punición.

Para comprobar que la intervención con el profesorado iba a ejercer influencia sobre el alumnado, se estudió también la motivación de estos últimos mediante el cuestionario (Cuestionario de Motivación para Estudiantes de Danza) cuya factorización arrojó tres agrupamientos posibles, denominados por los autores como "Percepción del Estilo del Profesor", "Sensación Propia" e "Interacción Motivacional".

Los datos derivados del alumnado demuestran que el cambio efectuado por los/as profesores/a del Grupo Experimental en su estilo docente son claramente percibidos por los alumnos/as; en consecuencia aumenta su motivación, así como la importancia que ellos mismos otorgan al trabajo motivacional en la danza.

Los resultados muestran que el alumnado puntúa más alto en la variable estilo del profesor después de que estos hayan asistido a las sesiones de intervención. Este cambio es tan relevante que la diferencia entre el antes $(M=28)$ y el después $(\mathrm{M}=30)$ es estadísticamente significativa $(t(2)=-2.05 ; \mathrm{p}=.047$; $\left.\mathrm{n}^{2}=.67\right)$. La sensación propia o dicho de otro modo, la variable que mide la motivación del alumnado también se ve aumentada con la formación de sus profesores aunque este cambio $(M$ pretest $=11 ; M$ postest $=11.6)$ no sea estadísticamente significativo $(t(2)=-1.01 ; \mathrm{p}=.32)$. En último lugar, la interacción motivacional aumentó sus valores medios de forma estadísticamente significativa en el grupo experimental ascendiendo de un valor de 14.1 antes de las sesiones a 14.6 después de éstas $\left(t(2)=-2.27 ; \mathrm{p}=.028 ; \mathrm{n}^{2}=.72\right)$.

Con el objetivo de comprobar que los cambios experimentados por los/as alumnos/as del profesorado que asistió a la formación no se debían a otras variables extrañas, se estudió de la misma forma al Grupo Control de alumnos. De este modo, la valoración que otorgan al estilo del profesor (percepción de estilo) disminuye su puntuación en la segunda recogida de datos $(M=29.7)$ en comparación con la primera $(M=34.4)$ siendo incluso esta discrepancia entre valores significativa $\left(t(1)=2.42 ; \mathrm{p}=.034 ; \mathrm{n}^{2}=.0 .85\right)$. Es decir, los alumnos y alumnas puntúan de forma más negativa el estilo del profesor cuando el curso académico está cerca de terminar y esto le sucede a un porcentaje significativo de estudiantes. En consonancia con lo anterior, la sensación propia motivacional también se ve reducida significativamente $(t(1)=2.34$; $\left.\mathrm{p}=.039 ; \mathrm{n}^{2}=.73\right)$ entre el primer momento en que se pasa el cuestionario alrededor de Febrero $(M=12.4)$ en comparación con la segunda vez que es alrededor de Mayo $(M=10.7)$. Por último, la interacción motivacional no experimenta ningún cambio significativo ( $p>.05)$ entre la primera vez que se recogieron los datos $(\mathrm{M}$ pre $=14.5)$ y la segunda $(\mathrm{M}$ post $=14.5)$.

Los resultados sugieren que existen diferencias en ambos grupos de alumnos; en el caso del Grupo Experimental dichos resultados pueden ser considerados positivos y son posiblemente un efecto derivado de la formación de sus profesores. Sin embargo, el Grupo Control aunque también presenta diferencias estadísticamente significativas, estos cambios po- 
seen un signo negativo, es decir, empeoran los niveles motivacionales y la percepción de estilo del profesor debido al natural curso académico. En este sentido, tanto la percepción del estilo del profesorado como la sensación propia motivacional descienden considerablemente entre los meses de Febrero y Mayo (final de curso) sugiriendo tal vez un declive generalizado de la motivación conforme se van acercando al final del curso académico.

\section{Discusión}

La intervención psicológica llevada a cabo con el profesorado, en la forma de sesiones de formación, ha repercutido positivamente sobre su estilo docente, mejorando la conducta instruccional -instrucciones generales e instrucciones técnicas ante los errores-incrementando las conductas de apoyo -conductas de reforzamiento y de ánimo contingente al error- $y$ disminuyendo las punitivas: castigo o punición e instrucciones técnicas punitivas.

Asimismo, el presente estudio ha mostrado los cambios producidos en la motivación del alumnado debido a la mejora en el estilo de enseńanza del profesorado tras asistir al período de formación. Dichos cambios hacen referencia a la valoración otorgada a las tres categorías que abarca el Cuestionario de Motivación para Estudiantes de Danza aplicado al alumnado: el estilo del profesor/a al dar las clases, la sensación motivacional que perciben de este estilo, y la importancia que dan al papel motivacional en la danza.

Estas fluctuaciones representan una muestra de los beneficios que ha producido la intervención psicológica en la actitud del profesorado. La variable instrucciones cambió significativamente después de las sesiones formativas, aumentando de forma significativa en todo el profesorado del grupo experimental. Este dato resulta destacable porque revela que se adquirieron unas estrategias útiles para relacionarse con el alumnado y, por tanto, la implicación del profesorado en las clases aumentó considerablemente.

El profesor deja entonces de ser una persona poco involucrada y aparentemente distante en clase, que sólo participa para corregir en determinados casos y de forma punitiva, para introducirse en el proceso de enseñanza sin personalizar los errores técnicos, utilizando ahora instrucciones generales acompañadas de instrucciones más concretas, emitidas con un tono positivo y con confianza hacia el alumnado. Cabe señalar que después de las charlas, un gran número de conductas de este tipo iban seguidas de reforzamiento positivo cuando el/la alumno/a ejecutaba sus movimientos de forma correcta. Esto explica también, el incremento de conductas de apoyo, una vez que se les ha formado sobre cómo ofrecerlas.

Es posible que la formación recibida por el profesorado le haya sensibilizado hacia las circunstancias en las que se desenvuelven los estudiantes, dándoles herramientas para instruir positivamente, y eso les motivará para enseñar de una forma diferente a la anterior. Cuando el profesorado, por lo general, está dispuesto a aprender para mejorar su relación con el alumnado y se le ofrecen patrones de conductas positivas, los incorporarán -tal y como se ha comprobado en este trabajo- a su repertorio conductual habitual, modificando en ocasiones aquello que no es coherente con lo aprendido.

Algo distinto ocurre en el Grupo Control, en el que la categoría Instrucciones no muestra diferencias significativas entre el antes y el después de las sesiones formativas. En cuanto a las otras categorías, la de Apoyo no sigue una tendencia clara entre ambos momentos y la de Punición tampoco refleja ningún cambio. Según esto, parece que las variaciones ocurridas en el Grupo Experimental eran más regulares, positivas y uniformes mientras que en el Grupo Control dependen en mayor medida de variables no controladas.

Por otro lado, se evaluaron los niveles motivacionales del alumnado para comprobar si ellos también se beneficiaban de la formación de sus profesores y para confirmar que el cambio esperado se había dado efectivamente a lo largo de los meses de formación y no estábamos siendo los investigadores víctima del conocido efecto de deseabilidad social; es decir, el profesor sabe cómo hay que instruir y de esta forma "actúa" de una forma artificial cuando se realiza la segunda observación.

Sin embargo, cuando se registran los cambios producidos en la motivación del alumnado del Grupo Experimental, cuyos profesores asistieron a las sesiones de técnicas motivacionales, se comprueba cómo aumenta la valoración que los estudiantes otorgan al estilo del profesor, siendo esta diferencia estadísticamente significativa. En cambio, el Grupo Control no refleja dicha discrepancia en el mismo sentido, es más se comprueba cómo la diferencia se produce exactamente en el sentido contrario, es decir empeorando tanto la percepción del estilo del profesorado como la sensación propia motivacional en la segunda observación. Esto se podría deber al cansancio producido por el momento del curso académico en el que se encontraban, ya que se realizó durante el mes de Mayo, cuando la mayoría se sienten más agotados física y psicológicamente. Este hecho parece importante para comprender cómo cambia la motivación natural de los alumnos y cómo pueden cambiar también los profesores en los diferentes momentos del año.

El tercer factor, denominado Interacción Motivacional, hace referencia a la relevancia que tienen trabajos como el realizado en este estudio con los bailarines y sus profesores, así como a la influencia que tiene la Psicología en el rendimiento de aquellos. En el Grupo Control no hay diferencias significativas entre el pretest y el postest, arrojando la misma puntuación en ambos momentos. Esto quiere decir que el hecho de ser evaluados por segunda vez -sin mediar ningún tipo de formación del profesorado entretanto- no produce por sí sólo una mejora en esos niveles. 
Este estudio permite demostrar el posible efecto positivo de las sesiones sobre el estilo del profesorado: éstos han sido capaces de invertir el hastío -hasta cierto punto natural- producido por el desarrollo del curso, mejorando la motivación del alumnado al final del mismo. Aunado con lo anterior, se aprecia también un cambio significativo en la interacción motivacional del Grupo Experimental, esto es, en la importancia que el alumnado considera que tiene la motivación en ellos mismos y en el profesorado de danza. A raíz de este resultado, se puede deducir que los estudiantes han percibido un cambio en la forma de dar las clases de sus profesores, mejorándose la motivación a lo largo del curso; al mismo tiempo se hacen conscientes de que ese cambio ha provocado una mejora en ellos mismos, en su rendimiento y en su agrado al asistir a clase. En conclusión, se podría entender que la mayoría de alumnos creen necesario este tipo de estudios y de trabajo con el profesorado y que así lo solicitan cuando se han beneficiado directamente de éste.

En general, los resultados certifican la influencia de las sesiones de formación tanto en la actitud del profesorado como en la motivación del alumnado. Como se puede deducir de lo anterior, se confirma también la estrecha conexión que existe entre la forma de instruir del profesorado y la consiguiente mejora de la motivación de sus estudiantes. Se podría concluir, en consecuencia, que los alumnos son extremadamente sensibles a la actitud del profesorado y que éste último tiene una gran responsabilidad en cómo se enfrentan diariamente sus alumnos a las clases, las ganas que tienen de trabajar $y$, por consiguiente, el esfuerzo que van a invertir en ellas. Como se ha comprobado, a mayor cantidad de Instrucciones positivas y de Apoyo y, por el contrario, menor Punición, el alumnado valorará de forma más positiva el estilo del profesorado y viceversa.

Los resultados del estudio permiten apoyar los obtenidos por, entre otros, Day, Elliot y Kington (2005), Firestone (1996), Graham (1996) y Louis (1998), acerca de la conveniencia de dar al profesorado pautas de acción que mejoren el funcionamiento de sus clases, la motivación de sus alumnos y las relaciones entre unos y otros. Dado el escaso número de investigaciones en psicología de la danza, el estudio de la motivación se hace necesario a causa de las características, especialmente duras, del trabajo diario de los bailarines.

Asimismo, se constatan evidencias similares a las de Thomas (1993), en cuanto a que el profesorado de danza verdaderamente efectivo es muy positivo, muestra apoyo y suministra al alumnado feedback con respecto a sus ejecuciones. Igualmente se coincide con autores como Black y Weiss (1992), Amorore y Horn (2000), o Rodríguez (2010), quienes encontraron, en diversos grupos de deportistas, que su motivación corre pareja con la percepción que éstos tienen del comportamiento instruccional de sus entrenadores.

Sin embargo, aunque se ha podido identificar un estilo docente que produce mayor motivación en el alumnado, así como la repercusión de las sesiones formativas -capaces incluso de contrarrestar el agotamiento producido por el curso académico- se deben tomar los resultados con cautela, puesto que la muestra de profesores no es tan amplia como para poder extrapolarlos con garantías. Para poder lograrlo, sería necesario que en futuras investigaciones se evaluara a un mayor número de profesores y alumnos, pudiéndose comprobar entonces, con mayor fiabilidad, la influencia que el predominio de ciertas conductas del profesorado puede tener en la motivación del alumnado.

Confiamos en que estos hallazgos y su traducción en los correspondientes planes formativos, contribuirán al desarrollo de la formación de los docentes de los Conservatorios de Danza y su repercusión se hará patente en la motivación, en el rendimiento y en la consecuente mejora de la calidad de vida del alumnado.

\section{Conclusiones}

Los resultados demuestran de alguna manera la repercusión positiva que tiene para todos, alumnos y profesores, el trabajo psicológico en danza. De esta forma, el presente estudio apoya parcialmente las hipótesis iniciales en cuanto que se ha mostrado un incremento en la motivación del alumnado de los profesores que acudieron a las charlas, siendo esta diferencia significativa para la escala de percepción de estilo del profesor e interacción motivacional. Paralelamente, se ha encontrado que el profesorado perteneciente al grupo experimental aumentó la frecuencia de conductas de apoyo e instrucciones y disminuyó las punitivas. Dicho cambio fue significativo para la conducta de instrucciones y mostró una tendencia clara pero no significativa en las conductas de apoyo y punición; por el contrario, esta tendencia no se ha observado en el grupo control.

Por tanto, se podría afirmar que en general los resultados apoyan los estudios de Brinson (1993) y Brinson y Dick (1996), sobre las mejoras que producen la intervención y la promoción de ciertas actitudes y conductas en este campo, a veces tan olvidado. En consecuencia, parece conveniente un plan de reciclaje periódico del profesorado de los centros de danza, introduciendo técnicas de formación como las descritas.

En definitiva, el ámbito de las enseñanzas artísticas necesita de los conocimientos psicológicos -eficaces en otros campos, como el deporte y la educación- para tratar con los problemas particulares que presentan y mejorar el rendimiento del alumnado. La formación del profesorado se considera imprescindible si nuestro objetivo es mejorar la formación y la calidad de vida de los bailarines, por lo que este tipo de intervenciones deberían ser habituales en un futuro próximo, al menos en aquellos Conservatorios y Escuelas de Danza 
donde la figura del psicólogo-orientador no esté establecida y donde tanto el profesorado como el alumnado, a menudo no saben ni a quién ni dónde acudir cuando necesitan asesoramiento.

\section{Referencias}

1. Amorore, A., y Horn, T. (2000). Intrinsic Motivation: Relationships with collegiate athletes' gender, scholarship status, and perceptions of their coaches' behavior. Journal of Sport and Exercise Psychology, 22, 63-84.

2. Ackard, D., Henderson, J., y Wonderlich, L. (2004). The associations between childhood dance participation and adult disordered eating and related psychopathology. Journal of Psychosomatic Research, 57(5), 485-490.

3. Black, S. J., y Weiss, R. (1992). The relationship among perceived coaching behaviours, perceptions of ability and motivation in competitive age group swimmers. Journal of Sport and Exercise Psychology, 14, 309-325.

4. Brinson, P. (1993). Training Tomorrow's Professional Dancers: The Papers of the 1993 Conference, London: Laban Centre

5. Brinson, P., y Dick, F. (1996). Fit to Dance? The Report of the National Inquiry into Dancers' Health and Injury, London: Calouste Gulbenkian Foundation.

6. Carter, C. S. (2005). Effects of formal dance training and education on student performance, perceived wellness, and self-concept in high school students. Humanities and Social Sciences, 65(8-A), 2906.

7. Caracuel, J. C., y García-Dantas, A. (2010). Danza, ejercicio físico y deporte: similitudes y diferencias. Comunicación presentada al VII Congreso Iberoamericano de Psicología. Oviedo, 20-24 de Julio.

8. Chappell, K. (2007). The dilemmas of teaching for creativity: Insights from expert specialist dance teachers. Thinking Skills and Creativity, 2(1), Apr, 39-56.

9. Day, C., Elliot, B., Kington, A. (2005). Reform, Standards and Teacher Identity: Challenges of Sustaining Commitment. Teaching and Teacher Education: An International Journal of Research and Studies, 21(5), 563577.

10. Firestone, W. (1996). Images of Teaching and Proposals for Reform: A Comparison of Ideas from Cognitive and Organizational Research. Educational Administration Quarterly, 32, 209-235.

11. García-Dantas, A., y Caracuel, J. C. (2009). Factores que influyen en el abandono del aprendizaje de la danza: un estudio en el Conservatorio Profesional de Danza de Sevilla. Comunicación presentada al XII Congreso Andaluz de Psicología de la Actividad Física y del Deporte. Jaén, 12-14 de Noviembre.
12. García-Dantas, A., y Caracuel, J. C. (2010). Competencia percibida en jóvenes bailarines: un estudio en el Conservatorio Profesional de Danza de Sevilla. Comunicación presentada al XII Congreso Nacional de Psicologia del Deporte y I Jornadas Internacionales de la AMPD. Madrid, 23 y 26 de Junio.

13. García-Dantas, A., y Caracuel, J. C. (2011). Psicología y danza. En, F. Arbinaga, y J.C. Caracuel, (compils.), 2011. Intervención psicológica en actividad fisica y deportes minoritarios (139-162). Madrid: Psimática.

14. Gordon, C. (2005). The critical and the appreciative attitudes to dance in education. Research in Dance Education, 6(1-2), 53-64.

15. Graham, K. C. (1996). Running ahead: Enhancing teacher commitment. Journal of Physical Education, Recreation and Dance, 67(1), 45-47.

16. Louis, K. S. (1998). Effects of teacher quality of work life in secondary schools on commitment and sense of efficacy. School Effectiveness and School Improvement, 9(1), 1-27.

17. López de la Llave, A. y Pérez Llantada, M. C. (2006). Psicología para intérpretes artísticos. Estrategias para la mejora técnica y profesional. Madrid: Thomson.

18. Marrero, J. (1993). Las teorías implícitas del profesorado: vínculo entre la cultura y la práctica de la enseñanza. En M.J. Rodrigo, A. Rodríguez y J. Marrero (Eds.). Las teorías implícitas. Madrid: Aprendizaje/Visor.

19. Rodríguez, M. P. (2010). Diseño, Aplicación y Evaluación de un programa de Intervención Psicológica en Equipos Juveniles de Fútbol. Tesis Doctoral inédita. Universidad Autónoma de Barcelona, Barcelona.

20. Sekulic, D., Peric, M., y Rodek, J. (2010). Substance use and misuse among professional ballet dancers. Substance Use \& Misuse, 45(9), 14201430.

21. Smith, F., Smoll, F. L., y Hunt, E.B. (1977). CBAS. A system for the behavioral assessment of athletic coaches. Research Quarterly, 48, 401-407.

22. Taylor, J., y Taylor, C. (2008). Psicología de la Danza. Madrid: Gaia Ediciones.

23. Thomas, B. (1993). Psychology and the Art of Positive Thinking. Bulletin of the Royal Academy of Dancing, June.

24. Wandzilak, T., y Ansorge, C. J. (1998). Comparison between selected practice and game behaviors of youth sport soccer coaches. Journal of Sport Behaviour, 11(2), 78-99. 


\section{CATEGORÍAS DEL REGISTRO OBSERVACIONAL}

CBAS (Smith, Smoll y Hunt, 1977) adaptado al Profesorado de Danza

\begin{tabular}{|c|c|c|}
\hline Alumnos/as & Profesor/a & \\
\hline \multirow{2}{*}{ Aciertos } & Reforzamiento+ & $\begin{array}{l}\text { Materiales: premios, sistema de Puntos. } \\
\text { Sociales: "muy bien"," estupendo", "bien hecho" }\end{array}$ \\
\hline & Utiliza como modelo & $\begin{array}{l}\text { Al que lo hace bien le utiliza como modelo para los demás, de una manera } \\
\text { positiva para el aprendizaje de todos }\end{array}$ \\
\hline \multirow{7}{*}{ Errores } & Instrucción Técnica + & $\begin{array}{l}\text { Corrige con actitud positiva, con ganas, sin ofender a nadie. Pone en } \\
\text { práctica habilidades sociales }\end{array}$ \\
\hline & Refuerza modelo + & Utiliza modelos para que aprendan los demás, evita competitividad \\
\hline & Ánimo contingente & $\begin{array}{l}\text { Le anima para que no se preocupe, quita importancia al error, apoya } \\
\text { fomentando confianza en el alumnado pasa nada, inténtalo" }\end{array}$ \\
\hline & Comparación con otros- & $\begin{array}{l}\text { Competitividad: "x lo hace bien, no como otros", clara división entre } \\
\text { los "listos y los torpes" }\end{array}$ \\
\hline & Estrategia de castigo & Excluye a los que lo hacen mal, sienta al que se equivoca, lo deja atrás \\
\hline & Instrucción técnica punitiva & Corrige de forma negativa, se enfada, "insultando" a los alumnos \\
\hline & Desánimo, desgana & $\begin{array}{l}\text { Conducta no verbal, desprecios, enfadado, agresivo, desgana, chantaje, } \\
\text { sin corregir, "no merece la pena" }\end{array}$ \\
\hline \multirow{5}{*}{$\begin{array}{l}\text { Conductas NO deseables } \\
\text { (hablar en clase, molestar a } \\
\text { otros compañeros, etc) }\end{array}$} & Refuerza modelo + & Se basa en el que lo hace bien, no competitivo \\
\hline & Instrucción sobre lo correcto & Debemos estar callados, hay que portarse bien porque... \\
\hline & Castigo & Idea una estrategia de castigo, por ej., "echarle de clase" \\
\hline & Prohibición & $\begin{array}{l}\text { Tiene reglas y se lo recuerda, lo prohíbe, se basa en lo que ha hecho } \\
\text { mal y no dice cómo se hace bien. Evita dialogar y no explica propósito } \\
\text { del por qué hay que hacerlo bien }\end{array}$ \\
\hline & Reprimenda & Le da la charla enfadado, disgustado, serio, desprecio \\
\hline \multirow{2}{*}{ Conductas generales } & Da ánimo general + & $\begin{array}{l}\text { Motiva a la clase, "vamos chicos", "asi se hace", "mis mejores bailarines", } \\
\text { "ánimo, vais a llegar lejos" }\end{array}$ \\
\hline & Instrucción técnica general & $\begin{array}{l}\text { Corrige de forma general, se dirige al grupo aunque el error sea individual, } \\
\text { evita personalización del error cuando es común }\end{array}$ \\
\hline
\end{tabular}

Nota: En cursivas las categorías positivas; sin cursivas las categorías negativas. 